\title{
Balancing building and maintenance costs in growing transport networks
}

\author{
Arianna Bottinelli, ${ }^{1}$ Rémi Louf, ${ }^{2}$ and Marco Gherardi ${ }^{3,4}$ \\ ${ }^{1}$ Mathematics Department, Uppsala University, Lägerhyddsvägen 1, Uppsala 75106, Sweden \\ ${ }^{2}$ Centre for Advanced Spatial Analysis, University College London, 90 Tottenham Court Road W1T4TJ London, United Kingdom \\ ${ }^{3}$ Sorbonne Universités, UPMC Univ Paris 06, UMR 7238, Computational and Quantitative Biology, \\ 15 rue de l'École de Médecine Paris, France \\ ${ }^{4}$ Dipartimento di Fisica, Università degli Studi di Milano, via Celoria 16, 20133 Milano, Italy \\ (Received 30 September 2016; revised manuscript received 14 September 2017; published 27 September 2017)
}

\begin{abstract}
The costs associated to the length of links impose unavoidable constraints to the growth of natural and artificial transport networks. When future network developments cannot be predicted, the costs of building and maintaining connections cannot be minimized simultaneously, requiring competing optimization mechanisms. Here, we study a one-parameter nonequilibrium model driven by an optimization functional, defined as the convex combination of building cost and maintenance cost. By varying the coefficient of the combination, the model interpolates between global and local length minimization, i.e., between minimum spanning trees and a local version known as dynamical minimum spanning trees. We show that cost balance within this ensemble of dynamical networks is a sufficient ingredient for the emergence of tradeoffs between the network's total length and transport efficiency, and of optimal strategies of construction. At the transition between two qualitatively different regimes, the dynamics builds up power-law distributed waiting times between global rearrangements, indicating a point of nonoptimality. Finally, we use our model as a framework to analyze empirical ant trail networks, showing its relevance as a null model for cost-constrained network formation.
\end{abstract}

DOI: 10.1103/PhysRevE.96.032316

\section{INTRODUCTION}

From roads, railways, and power grids, to ant trails, leaf veins, and blood vessels, transportation structures support the functions necessary to many natural and manmade systems [1-9]. Transport systems are typically represented as spatial networks, where nodes are distinct locations-such as cities or ant nests-and links are physical connections between these locations-such as roads or trails [10]. As transport networks are embedded in a metric space, the length of links is used to quantify the cost of building and maintaining the connections [11]. These costs pose an unavoidable constraint to transport networks, independent of their specific functions and intrinsically tied to their spatial nature. Together with the need for efficient transportation and for fault tolerance, costs affect the growth and the topology of transport networks, having profound impact on the systems that rely on them [11].

A great deal of theoretical and empirical research in physics, quantitative geography, and transport engineering has been devoted to understand how these constraints influence the evolution of transport networks and to identify minimal ingredients underlying the emergence of complex topologies [12-17]. The effects of competing design criteria have been explored: average shortest path versus link density [18] or total length [19], and total length versus synchronizability [20] or centrality [21]. Other models balance the length of newly added links with the gain in centrality [22] or efficiency [4], or analyze the costs and benefits entailed by their creation [23]. However, most of the existing models assume that (i) the network is static and/or constituted by a known set of nodes, (ii) it is either planned by a central authority, or the result of a completely self-organized process, and (iii) the length of a link is a proxy for both the costs of building and maintaining it [11]. Therefore, they neglect that (i) transport systems are typically built iteratively, often lack- ing information about future developments as these may be beyond the time horizon of planners [10,11]; (ii) due to such dynamic evolution, in long-lived infrastructures global planning has to compromise with local constraints and competing interests [10], and to alternate with local optimization processes; (iii) building costs and maintenance costs act on different time scales, constituting unavoidable constraints that cannot be optimized simultaneously.

These three aspects are strongly related. In a static scenario, the network of minimum length spanning a fixed set of nodes (the minimum spanning tree, MST) minimizes both maintenance and building costs [11]. In a dynamic setting, instead, when future node additions are not known ahead, or when the task of building links is partially delegated to local entities, these costs cannot be minimized simultaneously. On one side, building cost is minimized by iterating the local rule of "linking each new node to the closest node in the network." However, the obtained structure (called dynamical minimum spanning tree, dMST [22]) does not minimize the total length of the network [17], thus attaining a sub-optimal maintenance cost. On the other side, globally rearranging the network to a MST every time a node is added does minimize the total length, but it requires to destroy old links and rebuild new ones, increasing building cost. Moreover, maintenance costs must be sustained until links are abandoned or destroyed [24,25], constraining the network on a longer time scale.

In this paper, we address these open issues by formulating an out-of-equilibrium model for the growth of transport networks in the context where the position of new nodes cannot be predicted. By combining the global, centrally planned MST, and the local, decentralized dMST, the model explores the antagonism between the constraints associated with building and maintenance costs. (Such competing design goals have been suggested to be relevant also in nonspatial systems [26].) 


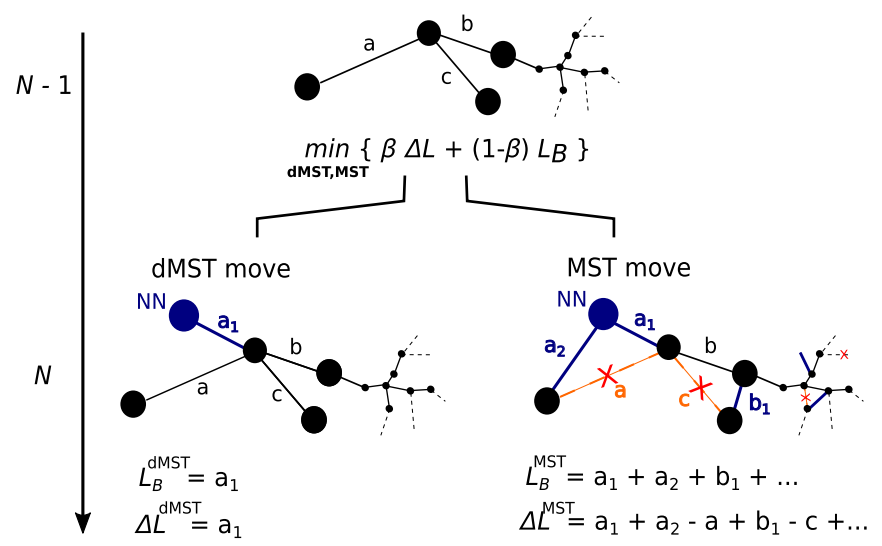

FIG. 1. At each time step, the model grows a network by adding a new node $(\mathrm{NN})$ at a random position. Depending on what move minimizes the convex combination of total built length $L_{B}$ and total length variation $\Delta L, \mathrm{NN}$ is either connected to the closest node in the network (dMST move) or the network is rewired to minimize the total length (MST move). In both cases only one link is added.

\section{MODEL}

Our model grows spatial networks starting from a single node and adding one node and one link at a time, so that the resulting networks are trees (see Fig. 1). (In real-world transport networks, fault tolerance is often achieved by relying on cycles [27,28]. Here we restrict to trees for simplicity.) Nodes appear with the flat measure on the unit square. When the $N$ th node at position $x_{N}$ is added to the existing nodes having positions $\left\{x_{1}, \ldots, x_{N-1}\right\}$, either it is linked to the closest node ("dMST move"), or a number of links are destroyed and rebuilt to obtain the (unique) MST spanning all nodes at positions $\left\{x_{1}, \ldots, x_{N}\right\}$ ("MST move"). The move is chosen such that the functional

$$
H(\beta, N)=\beta \Delta L(N)+(1-\beta) L_{\mathrm{B}}(N)
$$

is minimum. Here, $L_{\mathrm{B}}$ is the total length that needs to be built between $N-1$ and $N$, and $\Delta L$ is the variation in the network's total length $L$ (these are not equal, as $\Delta L$ includes negative contributions from the deleted links). For a spatial network $G$ built on $N$ nodes, the total length is defined as $L(G)=\sum_{e \in \mathcal{E}(G)} l_{e}$, where $e$ is a link belonging to $\mathcal{E}(G)$, the set of links of $G$, and $l_{e}$ is the Euclidean length of the link. Every time a node is added, $H$ is computed both for a MST and for a dMST move, then the MST move is performed if $H^{\mathrm{MST}}<H^{\mathrm{dMST}}$, and the dMST move otherwise. $\beta$ is the only parameter of the model, taking values in $[0,1]$ : we name it "strategy." Indeed, setting $\beta=0$ prioritizes the minimization of $L_{\mathrm{B}}$ (as expected if building costs are dominant), and the network grows only by local dMST moves. Conversely, $\beta=1$ minimizes $\Delta L$ (which is desirable when maintenance costs dominate), and the network is globally rewired to a MST at each step. When the two costs are comparable, intermediate values of $\beta$ account for both global and local length minimization and the model can alternate between MST and dMST moves. It is useful to express the growth condition $H^{\mathrm{MST}} \gtrless H^{\mathrm{dMST}}$ in terms of the sum of the lengths of newly built and newly destroyed links, $L_{\mathrm{B}}$ and $L_{\mathrm{D}}$, respectively, as

$$
L_{\mathrm{B}}^{\mathrm{MST}}-\beta L_{\mathrm{D}}^{\mathrm{MST}} \gtrless L_{\mathrm{B}}^{\mathrm{dMST}} .
$$

This is obtained by writing the explicit form of $H^{\mathrm{MST}}(N)$ and $H^{\mathrm{dMST}}(N)$, as shown in Appendix A 1.

\section{RESULTS}

For each value of $\beta$ from 0 to 1 (by steps of 0.02 ), we numerically grow 70 networks up to $N_{f}=1000$ nodes by the rules of the model. Results are averaged over these 70 networks. For each network, we measure the normalized Hamming distance, defined as the number of links that one has to create (and destroy) to turn the network $G$ into the MST spanning the same set of nodes (denoted $\hat{G}$ ), divided by the size of the network $N$ :

$$
d=\frac{|A(G)-A(\hat{G})|}{4 N},
$$

where $A$ indicates the adjacency matrix. This is the matrix extension of the well-known Hamming distance used in computer science to measure the distance between strings [29]. We will consider $d$ as a (random) function $d(\beta, N)$ of the strategy $\beta$ and the number of nodes $N$. The normalized Hamming distance identifies three classes of strategies separated by two transition points, $\beta_{1} \approx 0.45$ and $\beta_{2} \approx 0.82$ [Fig. 2(a)]. "MST-like" strategies $\left(\beta>\beta_{2}\right)$ grow networks at very small Hamming distance from the corresponding MST $(\beta=1)$. "dMST-like" strategies $\left(\beta<\beta_{1}\right)$ grow networks similar to the ones grown by iterating dMST moves only $(\beta=0)$. "Crossover" strategies $\left(\beta_{1}<\beta<\beta_{2}\right)$ smoothly interpolate between these two extremes. The phase boundaries and the value of $d(\beta, N)$ do not depend sensibly on network size after $N \approx 200$ [see Fig. 6 in Appendix A 2].

The existence of three classes is further confirmed by the scaling of the total length $L$ and efficiency $E$ with $\beta$, normalized by the corresponding MST values [Figs. 2(b) and 2(c)]. For a spatial network $G$ built on $N$ nodes, efficiency can be written as [30]

$$
E(G)=\frac{1}{N(N-1)} \sum_{i \neq j \in G} \frac{d_{i, j}^{e}}{d_{i, j}},
$$

where $\mathrm{i}$ and $\mathrm{j}$ are nodes in $G, d_{i, j}^{e}$ is their Euclidean distance, and $d_{i, j}$ is the length of the shortest path connecting them on the network $G$. It quantifies how quickly information and resources are exchanged over a transport network [30,31], and is often regarded as one of the main design goals in planning and building these networks $[8,12]$. It is known that maximizing efficiency competes with minimizing total length [32].

Interestingly, our approach reveals that balancing building and maintenance costs entails a tradeoff between total length and efficiency at large scale [Figs. 2(b) and 2(c)]. Notice that, in our model, this tradeoff depends on the size of the network, since the dMST is more efficient than the corresponding MST at large scale $(N \gtrsim 300)$, but less efficient at small scale $(N \lesssim 300)$ [see Fig. 6 in Appendix A 2]. Thus, our model suggests that the bias toward efficient transport observed in real networks may emerge under more general conditions, via 


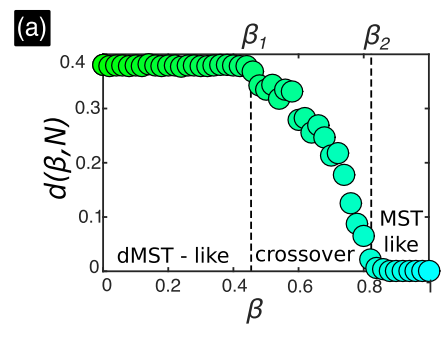

(b)

(d)

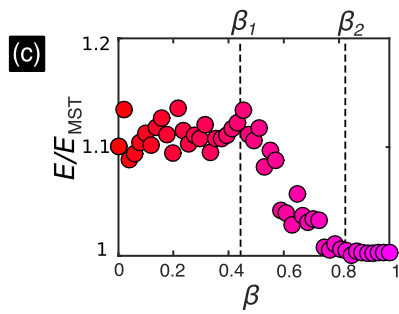

$-\mathrm{N}=1-300$

$\square \cdot \mathrm{N}=1-600$

$-N=1-1000$

... $\times \mathrm{N}=300-600$

$\nabla-\mathrm{N}=600-1000$
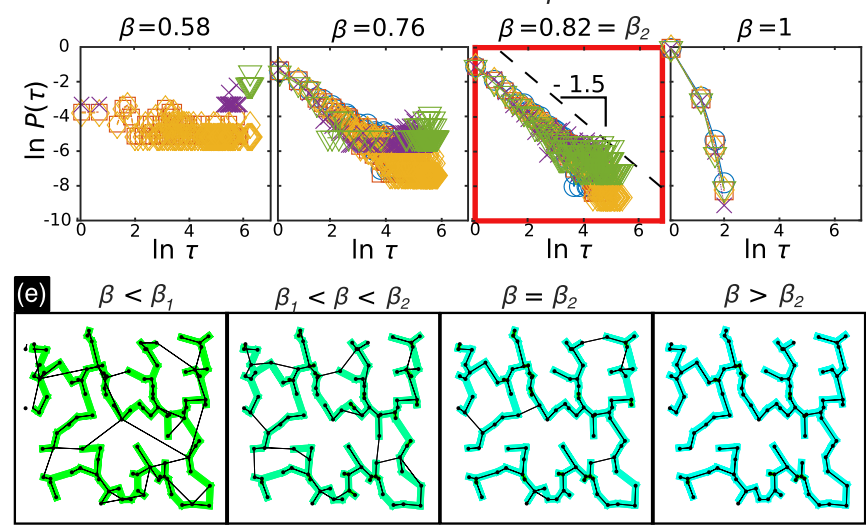

FIG. 2. Structural properties and evolution of networks grown with different strategies $\beta$. The normalized (a) Hamming distance, (b) total length, and (c) efficiency reveal three classes of strategies: MST-like, crossover, and dMST-like, separated by two transition points $\beta_{1}$ and $\beta_{2}$. (d) Probability distribution of the waiting time to the first MST move and between two consecutive MST moves $P(\tau)$ for different windows of network size. In $\beta_{2}, P(\tau)$ is a power law of exponent $\approx-1.5$ (highlighted box). (e) Realizations of the model (black thin line) for values of $\beta$ in the three classes of strategies and for $\beta=\beta_{2}$ on the same 50-nodes sequence (black dots), superposed to the corresponding MST (light bold line).

optimization of a function of length alone, and may also be dependent on the typical size of the observed network.

To better characterize the observed classes of strategies, we introduce the waiting time $\tau$, defined as the number of steps from $N=1$ to the first MST move, and then between two consecutive MST moves. Due to the nonstationarity of the process, the probability distribution function $P(\tau)$ of the waiting time depends not only on $\beta$, but also on the network's size $N$ [Fig. 2(d)]. Before $\beta_{1}$ the typical waiting times are larger than those attained by our simulations $\left(N_{f}=1000\right)$. Accordingly, the total length is never minimized through a MST move, and networks in this regime share only a few links with the corresponding MST, typically the shortest ones [Fig. 2(e), first box]. An estimate of $\beta_{1}$ can be obtained by using the condition for choosing a MST move $L_{\mathrm{B}}^{\mathrm{MST}}-\beta L_{\mathrm{D}}^{\mathrm{MST}}<$ $L_{\mathrm{B}}^{\mathrm{dMST}}$ [Eq. (2)], and assuming that, when $\beta$ is close to $\beta_{1}$ from above, a MST move destroys and re-builds most of the network's links [Fig. 2(e)]. The average length of a link in a MST of $N$ randomly placed nodes can be estimated as $\sqrt{1 / c N}$, where $c$ is some constant. Thus, $L_{B}^{\mathrm{MST}} \sim N \sqrt{1 / c N}=$ $\sqrt{N / c}$ and $L_{\mathrm{B}}^{\mathrm{dMST}} \sim \sqrt{1 / c N}$, while $L_{D}^{\mathrm{MST}} \sim \sum_{n=1}^{N} \sqrt{1 / c n} \sim$ $2 \sqrt{N / c}$ (See also Appendix A 3). The left-hand side of the growth condition Eq. (2) becomes $(1-2 \beta) \sqrt{N / c}$. Since $L_{\mathrm{B}}^{\mathrm{dMST}}$ goes to zero for large $N$, the condition for at least one MST move to occur in this limit becomes $\beta>1 / 2$, which is not far from the observed $\beta_{1} \approx 0.45$.

Equation (2) also suggests that, at the onset of the crossover regime, the occurrence of a MST event is tied to the destruction of long links to build short ones. This condition is likely to be achieved at large $N$, when the links built at the beginning of the process are likely to be very long $(\sim \sqrt{1 / c})$ with respect to the ones that would be found in a MST at the same network size $(\sim \sqrt{1 / c N})$. Once a MST rewiring has occurred, no long links are left, and $L_{\mathrm{B}}^{\mathrm{dMST}}$ tends to zero at increasing $N$, explaining why when $\beta$ is close to $\beta_{1}$ it is unlikely to observe more than one MST event. Accordingly, $P(\tau)$ shows that MST events are rare and happen typically at large network size [Fig. 2(d), first box]. Increasing $\beta$ reduces the relevance of long links for satisfying the MST condition; indeed, the probability of shorter waiting times increases at small network size, making MST events more likely [Fig. 2(d), second box]. In the MST-like phase $\left(\beta \gtrsim \beta_{2}\right)$, the growth condition is often satisfied, and $P(\tau)$ decays subpolynomially (exponentially for $\beta=1$ ) at all network sizes [Fig. 2(d), fourth box].

Remarkably, the dynamics displays long-range memory at the transition to the minimum-length phase $\beta_{2}$. Here $P(\tau)$ is a power law of exponent $\approx-1.5$ at all sizes [Fig. 2(d), third box], and the waiting time $\tau$ has no typical scale (except the cut-off) contrary to the MST-like and dMST-like phases. As a consequence, the occurrence of a MST event is highly unpredictable at $\beta_{2}$. Interestingly, -1.5 appears as a critical exponent in several models and empirical systems, e.g., in critical branching processes, self-organized criticality, firing neurons, priority queues, and human activity [33-35].

We analyze the long-term total cost of different growth strategies by means of three time-integrated quantities $\mathcal{L}_{\mathrm{B}}, \mathcal{L}_{\mathrm{D}}, \mathcal{L}_{\mathrm{M}}$, defined as

$$
\mathcal{L}_{*}\left(N_{f}, \beta\right)=\sum_{N=1}^{N_{f}} L_{*}(N, \beta) / \sum_{N=1}^{N_{f}} L_{*}(N, \beta=1) .
$$

These quantities measure how much length was built $(*=\mathrm{B})$, destroyed $(*=\mathrm{D})$, or maintained $(*=\mathrm{M})$ up to $N_{f}=1000$ by each strategy $\beta . L_{\mathrm{B}}(N, \beta)$ and $L_{\mathrm{D}}(N, \beta)$ are the instantaneous lengths built and destroyed between step $N-1$ and step $N$, and $L_{\mathrm{M}}(N, \beta)$ is the total length of the network at size $N$. All the measures are normalized by the values they take in a pure MST dynamics (i.e., at $\beta=1$ ) with the same realization of the point process.

In the simple scenario where the costs of maintenance and building per unit length have ratio $h$, the final cost of a network is given by $\mathcal{L}_{\mathrm{B}}+h \mathcal{L}_{\mathrm{M}}$. Plotting this total cost against $\beta$ produces a cost landscape for each value of $h$ [Fig. 3(a)]. Each cost landscape has an absolute minimum, which identifies the optimal strategy $\beta_{\text {opt }}(h)$ for the given ratio $h$. Notice that the location of the minimum is more well-defined than appears in the plot, where curves appear flat due to the extended $y$ axis, as can be better appreciated in Fig. 3(b). Interestingly, crossover 

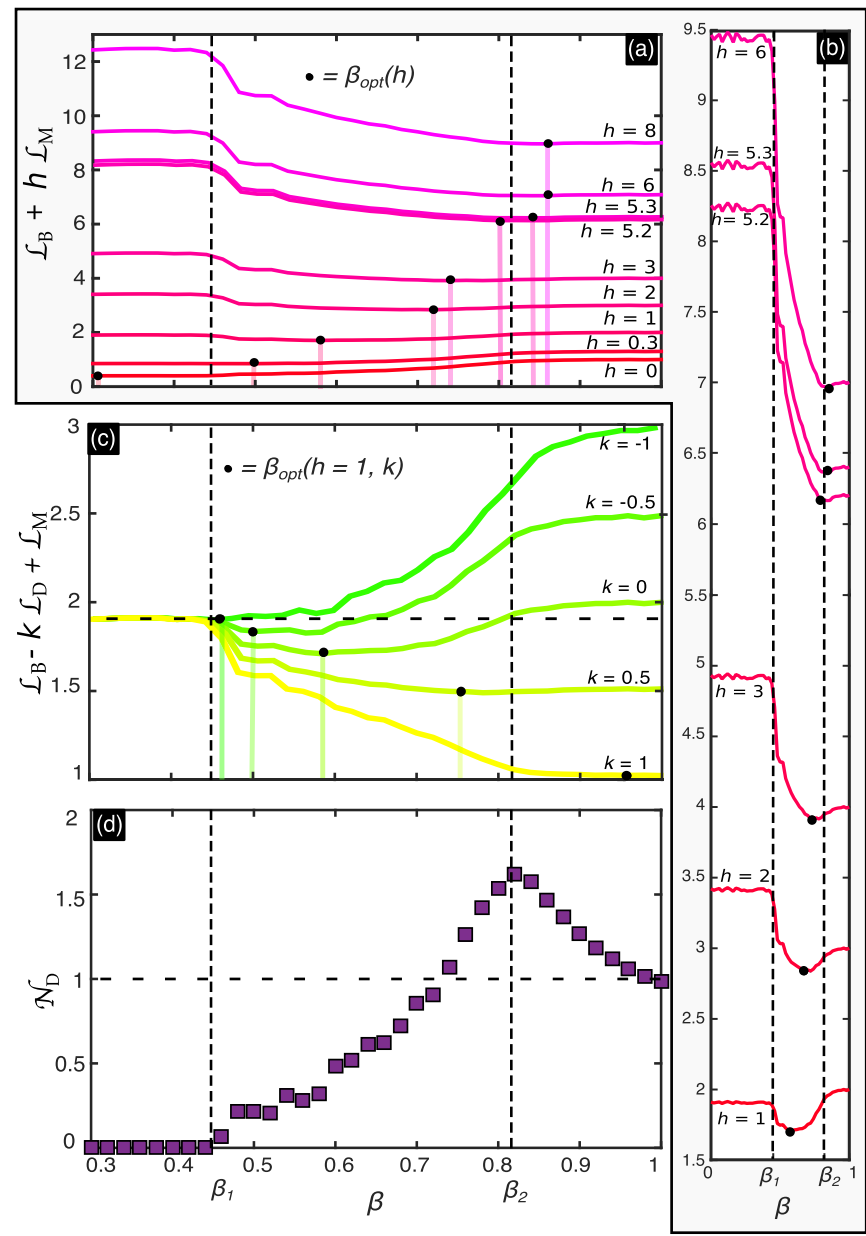

FIG. 3. The integrated (up to $N_{f}=1000$ ) total cost landscapes (solid lines) for (a) building $\left(\mathcal{L}_{\mathrm{B}}\right)$ and maintaining $\left(\mathcal{L}_{\mathrm{M}}\right)$, at different values of the ratio $h$ between their unit costs, (b) the same quantity with selected landscapes are plotted on same-scale axes to show the robustness of optimal strategies in the crossover regime; (c) building, maintaining, and destroying $\left(\mathcal{L}_{\mathrm{D}}\right)$ for $h=1 . k \in[-1,1]$ is the unit cost of destroying material (if $k<0$ ) or the advantage of recycling (if $k>0$ ). The minimum of each cost landscape (dots) is the optimal strategy $\beta_{\text {opt }}$ for the given value of $h$ and $k$, often belonging to the crossover regime. (d) The total number of links that are destroyed and rebuilt $\left(\mathcal{N}_{\mathrm{D}}\right)$ during the growth of the network is maximum in $\beta_{2}$, revealing high nonextensive costs. Strategies above the horizontal dashed line destroy more links than the pure strategy $\beta=1$.

strategies are optimal for a wide range of values of the ratio $h$ $(0.3 \lesssim h \lesssim 5.2)$, suggesting that these strategies would result optimal even in the presence of strong fluctuations or large uncertainties in the value of costs.

More complicated cost scenarios can be analyzed as well. For example, one may consider that building costs during a MST move may be reduced by recycling the material obtained from destroyed links. On the contrary, when recycling is not possible, disposing destroyed material may bear additional costs. In such scenarios, long-term costs can be expressed by means of the quantities defined above as $\mathcal{L}_{\mathrm{B}}+h \mathcal{L}_{\mathrm{M}}-k \mathcal{L}_{\mathrm{D}}$. The coefficient $k \in[-1,1]$ is the fraction of destroyed material that can either be recycled (if $k>0$ ) or bears additional disposal costs (if $k<0$ ). Also in this scenario, crossover strategies play an important role in minimizing the total costs of transport networks [Fig. 3(c), particular case of $h=1$ ], realizing nontrivial tradeoffs between the competing costs. It is worth noticing that the optimal strategy is in the crossover regime even when destroying is as expensive as building and maintaining $(k=-1)$, while MST-like strategies are optimal only when recycling strongly lowers the total cost.

All cost functions considered above are extensive in the length of the connections involved. However, lengthindependent costs may also be present, for instance, associated to setting up the sites for building and dismantling connections. More in general, we argue that building a transport network will also involve "fixed" costs that depend on the number of links modified at each step, regardless of their length. We quantify these nonextensive costs via the total number of links that were destroyed (and rebuilt) $\mathcal{N}_{\mathrm{D}}(N, \beta)=$ $\sum_{n=1}^{N} N_{\mathrm{D}}(n, \beta) / \sum_{n=1}^{N} N_{\mathrm{D}}(n, \beta=1) . N_{\mathrm{D}}$ is the number of links destroyed at each step, and the sum is normalized by the corresponding MST value, as in Eq. (5). Unexpectedly, $\beta_{2}$ is the strategy requiring the largest number of link deletions, and is therefore a point of nonoptimality in terms of fixed costs. Also, it is interesting to notice that strategies with $\beta \gtrsim 0.75$ require to destroy (and thus to rebuild) more links than in the pure MST strategy [Fig. 3(d)].

\section{APPLICATION TO ANT TRAIL NETWORKS}

In nature, a striking example of cost-constrained transport networks are the trails built by ant colonies to connect the nests that are iteratively built as the colony gets populous [3]. In this case, costs reflect the resources the colony has to spend to build and maintain the trails. These costs appear to vary for different species depending on the mechanisms underlying network formation, on environmental conditions, and on the kind of trails built $[9,17,32,36]$. For example, under laboratory conditions, the Argentine ant Linepithema humile builds pheromone-based trails, arranging them in globally optimized transport networks that resemble MSTs or even Steiner trees (minimum spanning trees where the set of nodes is allowed to be enlarged) [36] [Fig. 4(a), (i) and (ii)]. In the field, Argentine ants form instead more robust networks, possibly due to the necessity of resilience against predation and to environmental constraints [37]. While building costs are difficult to quantify for these pheromone-based networks, maintenance costs reflect the number of ants (and their energy consumption) needed to deposit the amount of pheromone necessary to maintain the trails [36].

The Australian meat ant Iridomyrmex purpureus builds physical trails that are kept clear of debris and vegetation and that can be up to 80 or 90 meters long [38] [Fig. 4(a), (iii) and (iv)]. These trails require a certain number of ants and an effort in terms of energy to cut the grass and to remove gravels and debris both at the time of building and during maintenance. Although there is no experimental evidence indicating whether meat ant colonies put more effort in building or in maintaining their trails, it appears that each newly built nest gets connected to the closest one in the colony, as happens in the dMST move [17]. It has also been observed that, during colony growth, meat ants may progressively abandon suboptimal connections and substitute them with shorter ones [38]. 


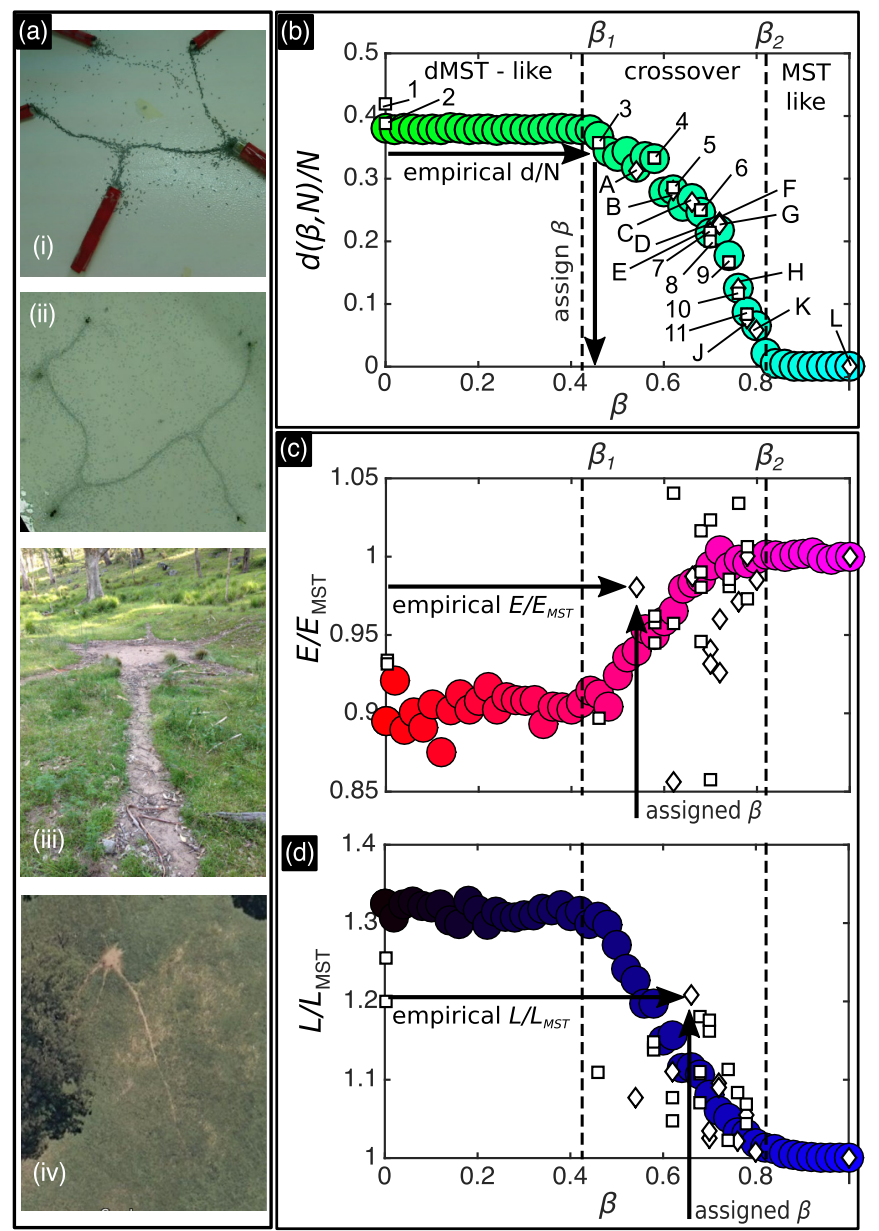

FIG. 4. (a) Examples of ant networks: (i), (ii) Argentine ants in laboratory conditions connect their nests through the shortest path. Courtesy of Tanya Latty. (iii) A Meat ant's nest with departing trails. Courtesy of Nathan Brown. (iv) Part of a meat ant's trail network from Google Earth. (b, c, d) Analysis of empirical networks through comparison with our model. Meat ants [39] are squares, other species [37,40-45] are diamonds, circles represent the model. (b) A value of $\beta$ is assigned to each ant network by comparing its rescaled Hamming distance with the prediction of our model. See Tables I and II. (c, d) For each ant network, the empirical values of the normalized efficiency (c) and normalized total length (d) are matched with the value of $\beta$ assigned as in panel (b), and plotted above the trend predicted by our model for $N=30$. Notice that networks with the same rescaled Hamming distance (and thus $\beta$ ) in (b) may have different values of efficiency and total length and thus correspond to distinct shapes in (c) and (d).

Altogether, these observations seem to suggest that building and maintenance costs have different impact on different species' construction strategies, also depending on environmental conditions. A better understanding may be obtained through manipulative experiments where, for example, the substrate where ants move is changed to substantially modify the relative cost of building and maintenance. The structural outcomes should then be observed and quantified at different ratios and for different species, likely making the whole experiment expensive and lengthy.
TABLE I. Summary of the structural properties of Meat ant's transportation networks from Ref. [39] [squares in Figs. 4(b), 4(c) and 4(d)]. First column: reference number. Ant networks with the same rescaled Hamming distance and $\beta$ correspond to the same reference number. Second column: number of nodes. Third column: number of links. Fourth column: rescaled hamming distance $d$. Fifth column: estimated $\beta$. Sixth column: normalized efficiency. Seventh column: normalized total length. * indicates that the network is a tree; i.e., $\mathcal{E}=N-1$.

\begin{tabular}{lcccccc}
\hline \hline & \multicolumn{5}{c}{$\square$ Meat ant data from Ref. [39]. } \\
\cline { 2 - 7 } Data & $N$ & $\mathcal{E}$ & $d$ & $\beta$ & $E / E_{\mathrm{MST}}$ & $L / L_{\mathrm{MST}}$ \\
\hline$[1]$ & 12 & $11^{*}$ & 0.42 & $\mathrm{ND}$ & 0.93 & 1.2 \\
{$[2]$} & 13 & $12^{*}$ & 0.38 & 0 & 0.93 & 1.26 \\
{$[3]$} & 14 & $14^{*}$ & 0.36 & 0.46 & 0.9 & 1.11 \\
{$[4]$} & 15 & 16 & 0.34 & 0.58 & 0.94 & 1.14 \\
& 15 & $14^{*}$ & 0.34 & 0.58 & 0.96 & 1.14 \\
& 12 & 11 & 0.34 & 0.58 & 0.96 & 1.15 \\
{$[5]$} & 14 & 14 & 0.29 & 0.62 & 0.95 & 1.08 \\
& 14 & 15 & 0.29 & 0.62 & 1.04 & 1.05 \\
{$[6]$} & 16 & 16 & 0.25 & 0.68 & 1.02 & 1.11 \\
& 16 & 17 & 0.25 & 0.68 & 0.95 & 1.07 \\
& 12 & $11^{*}$ & 0.25 & 0.68 & 0.99 & 0.18 \\
& 12 & 13 & 0.25 & 0.68 & 0.98 & 1.11 \\
{$[7]$} & 14 & 15 & 0.21 & 0.7 & 0.86 & 1.16 \\
{$[8]$} & 15 & 15 & 0.22 & 0.7 & 1.02 & 1.18 \\
{$[9]$} & 12 & 13 & 0.17 & 0.74 & 0.98 & 1.11 \\
& 12 & 12 & 0.17 & 0.74 & 0.99 & 1.02 \\
{$[10]$} & 17 & $18^{*}$ & 0.12 & 0.76 & 1.03 & 1.08 \\
{$[11]$} & 12 & $11^{*}$ & 0.08 & 0.78 & 0.97 & 1.04 \\
& 12 & 12 & 0.08 & 0.78 & 1 & 1.07 \\
\hline \hline & & & & & &
\end{tabular}

Thus, studies on ant transport networks have so far assumed building and maintenance costs to be proportional to the length of trails [9,17,31,32], and maintenance cost in particular to be proportional to the total length of the network [36]. More in general, robustness and efficiency are usually assumed to have a role in constraining network growth and topology [9,17,31,32]. Conversely, our model provides a minimal, general description of cost-constrained network growth where the topological tradeoff between total length and efficiency naturally arises from simply distinguishing between building and maintenance costs.

In what follows, we ask if our model can be utilized as a good "null-hypothesis" model in the attempt of better understanding the constraints acting on network construction in ants. We do not aim at a microscopic model of colony growth, but we use the model as a minimal description of the relevant optimization principles. Therefore, we do not intend to draw definite conclusions about the details of ants' behavior, but rather to give a minimal explanation of the observed empirical trends.

To this end, we collect 30 published networks constructed by the ant species Linepithema humile [37], Iridomyrmex purpureus [39-42], Formica lugubris [43,44], and Camponotus gigas [45] (Tables I and II). Ant networks are typically small (13 to 34 nodes), so one should be careful when analyzing them. For each network, we compute the rescaled Hamming distance by comparing the minimum-length skeleton (i.e., the 
TABLE II. Summary of the structural properties of ant networks data from Refs. [37,40-45] [diamonds in Figs. 4(b), 4(c) and 4(d)]. First column: reference in the figure and reference in the bibliography. Other columns as in Table I. * indicates that the network is a tree; i.e., $\mathcal{E}=N-1$.

\begin{tabular}{lcccccc}
\hline \hline & \multicolumn{6}{c}{$\diamond$ Other data from Refs. [37,40-45]. } \\
\cline { 2 - 7 } Data & $N$ & $\mathcal{E}$ & $d$ & $\beta$ & $E / E_{\mathrm{MST}}$ & $L / L_{\mathrm{MST}}$ \\
\hline A [37] & 16 & 18 & 0.31 & 0.54 & 0.98 & 1.07 \\
B [40] & 32 & 38 & 0.28 & 0.62 & 0.86 & 1.11 \\
C [44] & 19 & 24 & 0.26 & 0.66 & 0.99 & 1.21 \\
D [44] & 14 & 15 & 0.21 & 0.7 & 0.93 & 1.03 \\
E [44] & 14 & 16 & 0.21 & 0.7 & 0.94 & 1.03 \\
F [41] & 13 & $12^{*}$ & 0.23 & 0.72 & 0.96 & 1.1 \\
G [44] & 22 & $21^{*}$ & 0.23 & 0.72 & 0.93 & 1.09 \\
H [45] & 24 & 28 & 0.13 & 0.76 & 0.97 & 1.02 \\
J [42] & 13 & 13 & 0.08 & 0.78 & 1 & 1.05 \\
K [43] & 34 & 34 & 0.06 & 0.8 & 0.99 & 1 \\
L [44] & 13 & $12 *$ & 0 & 1 & 1 & 1 \\
\hline \hline
\end{tabular}

minimum spanning tree on the original network) with the MST built on the same set of nodes, and dividing by the size of the network [as prescribed by Eq. (3)]. Then, we assign a value of $\beta$ to each empirical network by comparing the obtained value of $d$ with the trend predicted by our model [Tables I and II, third and fourth columns, and Fig. 4(b)]. To test the compatibility between empirical structural properties and the value of $\beta$ assigned through our model, for each minimumlength skeleton we compute the normalized efficiency and total length (Tables I and II, last two columns). For each ant network we thus have two points, $\left(\beta, E / E_{\mathrm{MST}}\right)$ and $\left(\beta, L / L_{\mathrm{MST}}\right)$. Superposing them to the trends for $E / E_{\mathrm{MST}}$ and $L / L_{\mathrm{MST}}$ predicted by our model at small network size $(N=30)$ [Fig. 4(c)(d)] allows us to test whether $\beta$ alone can predict the structural properties of real transport networks. Finally, we apply the same procedure to randomized ant networks to have a benchmark for our analysis (Fig. 6). Randomized networks are obtained in $4 \mathcal{E}$ rewiring steps (where $\mathcal{E}$ is the number of links) starting from the adjacency matrix of empirical networks, and have the same set of degrees.
Figures 4 and 5 allow us to draw three main observations. First, the rescaled distance $d$ from the corresponding MST departs from the MST at most as much as the typical dMSTs $(d \approx 0.38)$ [Fig. 4(b)] in all analyzed trail networks (the only outlier is off by only a few percents). This was not to be expected a priori since, for example, in randomized networks the rescaled Hamming distance is systematically larger than the maximum value obtained by our model [Fig. 5(a)]. As a consequence, it is not possible to assign a strategy $\beta$ to most random networks, which reasonably reflects the fact that random networks are not cost-constrained. Second, for 27 colonies out of 30 , the estimated strategy $\beta$ is in the crossover regime [Fig. 4(b)]. Third, empirical networks tend to cluster around our model (except for two out-layers) for both efficiency and total length [Figs. 4(c) and 4(d)]. Of course, it is likely that Hamming distance and distance in length to some reference model are correlated in any network, i.e., networks that are dissimilar from a given network in terms of Hamming distance will be dissimilar also in terms of total length. However, the figure points out that empirical ant networks display a specific (albeit possibly null) relationship between total length and efficiency, which is well captured by the model and summarized by the assigned $\beta$ value.

\section{DISCUSSION}

Albeit simple, our interpolating model presents a rich behavior, providing a general framework to understand the competing nature of construction and maintenance costs in the context of spatial networks. In doing so, it addresses the interplay of central planning and local growth that characterizes the evolution of many manmade transport networks, offering insights into the long-term outcome of different construction strategies. Intermediate growth strategies are optimal in many cost scenarios, as they minimize the long-term total costs entailed by the infrastructure. Moreover, we showed that balancing competing costs is a minimal sufficient ingredient for the emergence of the tradeoff between the total length of the network and its transport efficiency, which is usually explained by more system-specific principles. Finally, the model displays a transition point with diverging characteristic time, similarly to the phenomenon of critical slowing down close to phase
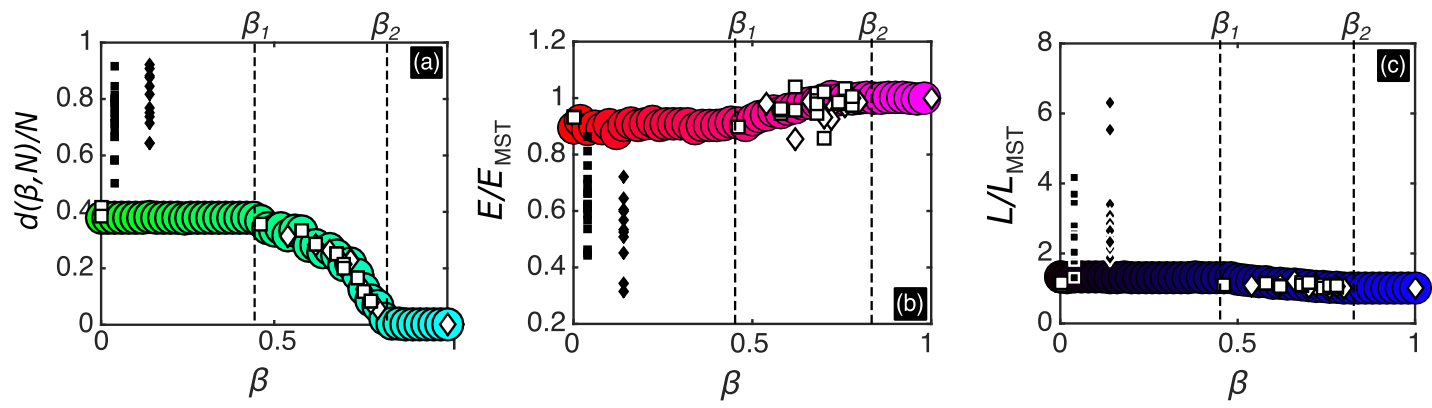

Legend: $\diamond \square$ empirical networks;

randomized networks;

model.

FIG. 5. Randomized network analysis. (a) The Hamming distance of randomized networks from the corresponding MST is larger than 0.5 with large probability, thus it is not possible to assign them a value of $\beta$. In this plot and the following ones they are plotted at arbitrary values (full shapes) and together with original ant data (empty shapes) and our model (circles) for comparison. Also, (b) efficiency and (c) total length of randomized networks assume values not compatible with ant data and with the model's predictions. 
transitions in statistical mechanics, which maximizes the long-term number of links rewired.

A key premise in the formulation of the model is that the position of new nodes is not known beforehand. If the time scale of the arrival of new nodes is much larger than that of the transport processes on the network itself, then each new node needs to be connected before the position of successive nodes can be taken into account. A possible example in human systems is the evolution of bus routes $[46,47]$. New areas can be quickly connected by adding further segments to bus lines stopping nearby (dMST move). However, if the whole network becomes suboptimal in terms of running costs, it may become necessary to redesign it globally (MST move). Our model suggests there may be an optimal re-organization strategy that minimizes the total costs entailed by the transport network.

Model-enabled analysis of ant trail networks data further proves the potential of the framework. The Hamming distances between the empirical networks and minimum spanning trees connecting the nests lie in the range predicted by a pure cost-optimization principle. This indicates that cost is a fundamental constraint in biological network formation, and that the structure of ant networks could in principle be explained by a cost optimization paradigm alone.

The fact the estimated strategy is in the crossover regime for most empirical networks seems to suggest that both maintenance and building costs may be relevant in constraining the growth of ant networks. However, the distribution of their rescaled distances in the interval $[0,0.4]$ does not appear to be substantially different from the distribution of a set of random numbers between the same extremes. This, together with the observation that, in our model, the optimal strategy is in the crossover regime for large fluctuations in the ratio of building and maintenance unit costs $(h)$ [Fig. 3(a)], prevents us from drawing definitive conclusions on whether $\beta$ reflects the actual balance between building and maintenance costs in these networks. Testing this hypothesis would require knowing the growth history of empirical networks, which is a nontrivial information in the case of ant networks, but may be easier to retrieve for some manmade transport networks.

There is semiquantitative agreement between model and data on the relation between the efficiency and the total length of empirical networks and its departure from a MST. So far, such a relationship has been explained and modelized as an explicit tradeoff between these two quantities [17,32]. However, the compatibility between the empirical values of efficiency and total length with the values predicted by our model (which is even more evident when comparing with the same values for randomized networks [Figs. 5(b) and 5(c)]), seems to support our hypothesis that the balance between efficiency and total length characterizing most empirical ant networks [32] may be explained by cost constraints alone.

Finally, we do not observe a clear distinction between different ant species in any of the analyzed plots. This may be due to the fact that empirical ant networks are small (between 13 and 34 nodes), and thus subject to strong fluctuations, but it could also be an indication that different species may share common underlying building principles and optimization strategies.

Drawing a definite conclusion on the biological meaning of $\beta$ as well as on the dominance of building and maintenance costs in shaping the structural properties of transport networks requires further investigation. However, the comparison between empirical and randomized networks supports the validity of the model as a null description relevant for cost-constrained networks. The value of $\beta$ alone (obtained from a purely topological quantity) gives a null prediction for metric quantities, such as efficiency and total length. In this regard, our model is a simple null benchmark that may be helpful in bringing out any positive phenomenology from the data. Moreover, it provides a new perspective to understand and analyze the growth of transport networks in diverse contexts. Altogether, our results advocate that both this global/local dichotomy, as well as the building and maintenance one, should be taken into account in the analysis and modelization of transport networks.

\section{ACKNOWLEDGMENTS}

We thank D. J. T. Sumpter, J. L. Silverberg, J. L. Denebourg, and M. Cosentino Lagomarsino for constructive feedback and stimulating discussions. We thank T. Latty and N. Brown for the pictures in Fig. 4. A.B. acknowledges funding from the Centre for Interdisciplinary Mathematics (CIM), Uppsala University.

\section{APPENDIX}

\section{Derivation of the growth condition}

Starting from Eq. (1) we can write $H^{\mathrm{MST}}(N)=$ $\beta\left(L^{\mathrm{MST}}(N)-L(N-1, \beta)\right)+(1-\beta) L_{B}^{\mathrm{MST}}(N, \beta) \quad$ and $H^{\mathrm{dMST}}(N)=L_{B}^{\mathrm{dMST}}(N) . L^{\mathrm{MST}}(N)$ is the total length of the minimum spanning tree at size N. $L(N-1, \beta)$ is the total length of the network at size $N-1$ and depends on both $N$ and $\beta$, as well as $L_{B}^{\mathrm{MST}}(N, \beta)$, that is the total length that needs to be built to get a MST at size $N$ from the network at size $N-1 . L_{B}^{\mathrm{dMST}}(N)$ is the length of the link that will connect the new node to its closest neighbor according to the dMST prescription. The total length of the MST at size $\mathrm{N}$ can be written as $\quad L^{\mathrm{MST}}(N)=L(N-1, \beta)+L_{B}^{\mathrm{MST}}(N, \beta)-L_{D}^{\mathrm{MST}}(N, \beta)$. $L_{D}^{\mathrm{MST}}(N, \beta)$ is the sum of the lengths of the links that need to be destroyed during a global MST rewiring. Thus, the equation governing the growth process can be written in a more compact fashion as

$$
L_{\mathrm{B}}^{\mathrm{MST}}-\beta L_{\mathrm{D}}^{\mathrm{MST}} \gtrless L_{\mathrm{B}}^{\mathrm{dMST}} .
$$

\section{Scaling of the structural properties with network size $N$}

At all values of $\beta$, the Hamming distance $d=(\mid A(G)-$ $A(\hat{G}) \mid) / 4 N$ is a linear increasing function of network size [Fig. 6(a)]. After a short transient $(N \sim 100)$, the rescaled hamming distance $d=\mathrm{d} / N$ is constant with respect to network size $N$, and can be used as a size-independent measure of the length optimization deriving from a certain strategy $\beta$.

The scaling of the total length as a function of $N$ at different values of $\beta$ is shown in Fig. 6(b). At each value of $\beta$, the total length scales as $\sqrt{N}$ multiplied by a constant that depends on $\beta$, as estimated in Appendix A 3. When divided by the total length of the corresponding MST (built on the same set of 

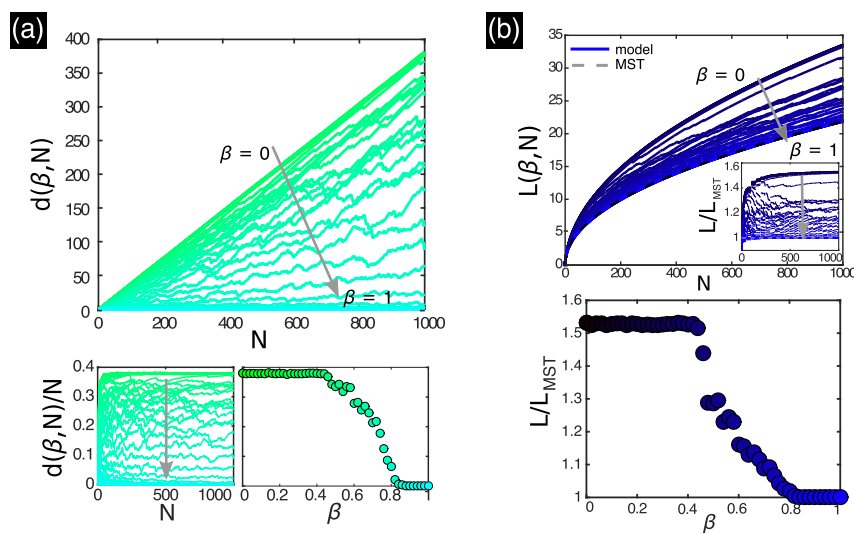

(c)

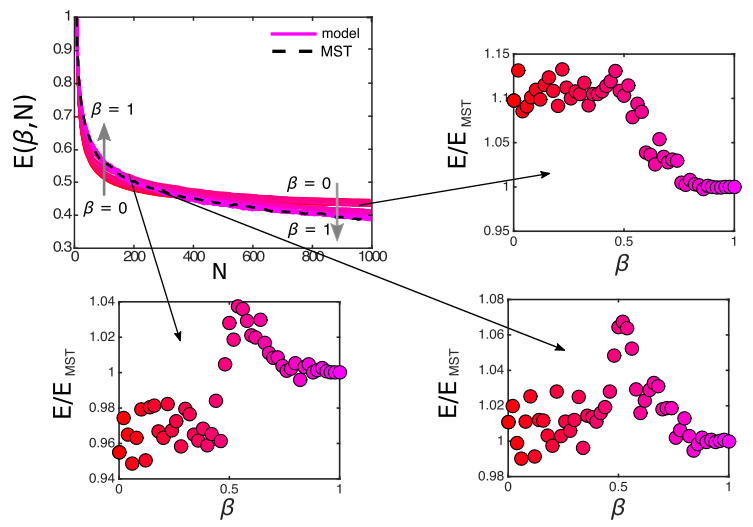

FIG. 6. Structural properties of the transport networks built by our model as a function of network size $N$ and of the strategy $\beta$ (colors online). Full lines represent our model at different values of $\beta$. Arrows indicate increasing values of $\beta$. Dashed lines corresponds to the MST built on the same set of nodes. (a) Top: The Hamming distance as a function of $N$ at different values of $\beta \in[0,1]$. Bottom: the rescaled Hamming distance as a function of $\mathrm{N}$ (left) and $\beta$ (right) for $N=1000$. (b) Top: total network length as a function of $N$ for different values of $\beta$, and rescaled by the length of the MST built on the same set of nodes (inset). Bottom: The rescaled total length as a function of $\beta$ at $N=1000$. (c) Top: Efficiency as a function of $N$ at different values of $\beta$. Smaller plots: Efficiency normalized by the corresponding MST value during three growth stages of the network at $N=200, N=350$, and $N=1000$ (counterclockwise). nodes), $L / L_{\mathrm{MST}}(N)$ fluctuates around a constant value after $N \sim 250$ for all values of $\beta$. To summarize the asymptotical behavior of the total length of the networks built by our model we take the rescaled total length $L / L_{\mathrm{MST}}$ at $N=1000$ and plot it against $\beta$.

Finally, the efficiency of our transport networks shows a different behavior depending on network size [Fig. 6(c)]. When the network is small $(N \sim 200)$ the dMST is less efficient than the corresponding MST. The ratio converges to 1 with increasing $N$. Around $N \sim 350$ the efficiency of the networks produced by our model is very close to the efficiency of a MST. Interestingly, the Hamming distance shows that the same efficiency is achieved by different network structures. At large network size, the rescaled efficiency shows the same three phases observed in the rescaled Hamming distance and in $L / L_{\mathrm{MST}}$, with low values of $\beta$ corresponding to highly efficient networks.

\section{Scaling of the mean total length with network size $N$}

For a network built on a random set of $N$ nodes the total length can be written as $L=\mathcal{E}\langle r\rangle$, where $\mathcal{E}$ is the number of links and $\langle r\rangle$ is the average length of links. For a Poisson distribution in $2 \mathrm{D}$ the distance between two first nearest neighbors is $\left\langle r_{1}\right\rangle=\frac{1}{2 \sqrt{\rho}} \rho=N / W$ is the density of points in the area $W$. For a tree $\mathcal{E}=N-1$, therefore,

$$
L^{\mathrm{MST}}=(N-1) \frac{1}{2 \sqrt{\rho}} \sim \frac{\sqrt{W}}{2} \sqrt{N} .
$$

The building prescription of the dMST implies that every new node is linked to its first neighbor iteratively, so it seems reasonable that

$$
L^{\mathrm{dMST}}=\sum_{i=1}^{N} \frac{\sqrt{W}}{2 \sqrt{i}}=\frac{\sqrt{W}}{2} H_{N}^{\left(\frac{1}{2}\right)} \sim \sqrt{W} \sqrt{N} .
$$

$H_{N}^{\left(\frac{1}{2}\right)}$ is the generalized harmonic number: $H_{N}^{\left(\frac{1}{2}\right)}=2 \sqrt{N}+$ $\zeta(1 / 2)+\ldots$, and $\zeta$ is the Riemann Zeta function. At the first order $L_{\mathrm{TOT}}^{\mathrm{dMST}} \sim \sqrt{W} \sqrt{N}$. Therefore, the total length of the MST and dMST have the same scaling as a function of $N$, what changes is a constant.
[1] H. Ronellenfitsch and E. Katifori, Phys. Rev. Lett. 117, 138301 (2016).

[2] A. Tero, S. Takagi, T. Saigusa, K. Ito, D. P. Bebber, M. D. Fricker, K. Yumiki, R. Kobayashi, and T. Nakagaki, Science 327, 439 (2010).

[3] A. Perna, B. Granovskiy, S. Garnier, S. C. Nicolis, M. Labédan, G. Theraulaz, V. Fourcassié, and D. J. T. Sumpter, PLoS Comput. Biol. 8, e1002592 (2012).

[4] M. T. Gastner and M. E. Newman, J. Stat. Mech.: Theory Exp. (2006) P01015.

[5] J. Buhl, J. Gautrais, N. Reeves, R. Solé, S. Valverde, P. Kuntz, and G. Theraulaz, Eur. Phys. J. B-Cond. Matter Complex Syst. 49, 513 (2006).
[6] S. P. Gorman and R. Kulkarni, Environ. Plan. B: Plan. Design 31, 273 (2004)

[7] G. Serini, D. Ambrosi, E. Giraudo, A. Gamba, L. Preziosi, and F. Bussolino, EMBO J. 22, 1771 (2003).

[8] D. P. Bebber, J. Hynes, P. R. Darrah, L. Boddy, and M. D. Fricker, Proc. Roy. Soc. B: Biol. Sci. 274, 2307 (2007).

[9] J. Buhl, K. Hicks, E. R. Miller, S. Persey, O. Alinvi, and D. J. Sumpter, Behav. Ecol. Sociobiol. 63, 451 (2009).

[10] F. Xie and D. Levinson, Netw. Spatial Econ. 9, 291 (2009).

[11] M. Barthélemy, Phys. Rep. 499, 1 (2011).

[12] M. T. Gastner and M. E. J. Newman, Phys. Rev. E 74, 016117 (2006).

[13] J. Banavar, A. Maritan, and A. Rinaldo, Nature 399, 130 (1999). 
[14] A. Maritan, F. Colaiori, A. Flammini, M. Cieplak, and J. Banavar, Science 272, 984 (1996).

[15] R. Guimerà, A. Díaz-Guilera, F. Vega-Redondo, A. Cabrales, and A. Arenas, Phys. Rev. Lett. 89, 248701 (2002).

[16] V. Colizza, J. R. Banavar, A. Maritan, and A. Rinaldo, Phys. Rev. Lett. 92, 198701 (2004).

[17] A. Bottinelli, E. van Wilgenburg, D. J. T. Sumpter, and T. Latty, J. Roy. Soc. Interf. 12, 20150780 (2015).

[18] R. Ferrer i Cancho and R. Solé, Statistical Mechanics of Complex Networks, Lecture Notes in Physics Vol. 625 (Springer, Berlin, 2003), pp. 114-125.

[19] M. Brede, Phys. Rev. E 81, 066104 (2010).

[20] M. Brede, Phys. Rev. E 81, 025202(R) (2010).

[21] M. Barthélemy and A. Flammini, J. Stat. Mech. (2006) L07002.

[22] A. Fabrikant, E. Koutsoupias, and C. H. Papadimitriou, Automata, Languages and Programming, Lecture Notes in Computer Science Vol. 2380 (Springer, Berlin, 2002), pp. 110-122.

[23] R. Louf, P. Jensen, and M. Barthelemy, Proc. Natl. Acad. Sci. USA 110, 8824 (2013).

[24] P. E. O'Sullivan, Transport Policy: Geographic, Economic, and Planning Aspects (Rowman and Littlefield, Lanham, MD, 1980).

[25] J. H. Taplin, M. Qui, V. K. Salim, and R. Han, Cost-benefit Analysis and Evolutionary Computing: Optimal Scheduling of Interactive Road Projects (Edward Elgar Publishing, Cheltenham, UK, 2005).

[26] S. Mandrà, M. C. Lagomarsino, and M. Gherardi, Phys. Rev. E 90, 032805 (2014)

[27] V. H. Louzada, F. Daolio, H. J. Herrmann, and M. Tomassini, Propagation Phenomena in Real World Networks 85, 215 (2015).

[28] R. Albert, H. Jeong, and A. Barabási, Nature 406, 378 (2000).
[29] R. W. Hamming, Bell Syst. Tech. J. 29, 147 (1950).

[30] V. Latora and M. Marchiori, Phys. Rev. Lett. 87, 198701 (2001).

[31] Z. Cook, D. W. Franks, and E. J. Robinson, Behav. Ecol. Sociobiol. 68, 509 (2014).

[32] G. Cabanes, E. van Wilgenburg, M. Beekman, and T. Latty, Behav. Ecol. 26, 223 (2014).

[33] S. Zapperi, K. B. Lauritsen, and H. E. Stanley, Phys. Rev. Lett. 75, 4071 (1995).

[34] N. Friedman, S. Ito, B. A. W. Brinkman, M. Shimono, R. E. Lee DeVille, K. A. Dahmen, J. M. Beggs, and T. C. Butler, Phys. Rev. Lett. 108, 208102 (2012).

[35] A. Vázquez, J. G. Oliveira, Z. Dezsö, K.-I. Goh, I. Kondor, and A.-L. Barabási, Phys. Rev. E 73, 036127 (2006).

[36] T. Latty, K. Ramsch, K. Ito, T. Nakagaki, D. J. Sumpter, M. Middendorf, and M. Beekman, J. Roy. Soc. Interf. 8, 1298 (2011).

[37] N. Heller, K. Ingram, and D. Gordon, Insectes Sociaux 55, 397 (2008).

[38] T. Greaves and R. D. Hughes, Austral. J. Entomol. 13, 329 (1974).

[39] E. Van Wilgenburg and M. Elgar, Insectes sociaux 54, 5 (2007).

[40] J. Holt, J. Trop. Ecol. 6, 379 (1990).

[41] A. N. Andersen and A. D. Patel, Oecologia 98, 15 (1994).

[42] J. McIver, Insectes Sociaux 38, 129 (1991).

[43] G. Boudjema, G. Lempérière, M. Deschamps-Cottin, and D. G. Molland, Ecol. Model. 190, 147 (2006).

[44] S. Ellis, D. W. Franks, and E. J. Robinson, Behav. Ecol. 25, 1183 (2014).

[45] M. Pfeiffer and K. Linsenmair, Insectes Sociaux 47, 123 (2000).

[46] J. Dodson, P. Mees, J. Stone, and M. Burke, Issues Paper 15, (2011).

[47] S. Sekhar, W. L. Yue, and M. Taylor, Int. J. Urban Sci. 8, 28 (2004). 